\title{
DUODENAL HAMARTOMA - A CASE REPORT AND REVIEW OF THE LITERATURE
}

\author{
N. Stefanova*, S. Spasova, M. Tzaneva \\ Department of General and Clinical Pathology, Forensic Sience and Deontology, Medical University - \\ Varna, Bulgaria
}

\begin{abstract}
Brunner's gland hamartoma is a rare benign tumor of the duodenum - a single pedunculated polyp. Usually asymptomatic and discovered incidentally, these lesions may manifest as a cause of duodenal obstruction or upper gastrointestinal hemorrhage and require surgical excision.

The aim of this study is to report a case of 46-year-old male patient presenting with abdominal discomfort. His past medical history was unremarkable. Fibrogastroduodenoscopy revealed a polyp of the duodenum sized $8 \mathrm{~mm}$ that was resected. Histological examination showed duodenal mucosa with hyperplasic villous structures and crypts with partial stomach epithelium, forming papillary proliferations. In the submucosa there were hyperplastic Brunner glands, some of them with cystic transformation, hyperplasia of tubules and villi lined by pseudostratified epithelium with reduction of the mucous secretion. Muscularis mucosae was with arborization of adipocytes. In the chorion there were a hyperplasia of smooth muscle cells and a lot of adipocytes. Immunostaining for MUC5 was positive in the metaplastic stomach epithelium while MUC2 was positively expressed only in the goblet cells. CD10 was positive in the apical part of the surface duodenal epithelium.

Several case reports have described these lesion as non - dysplastic and entirely benign. This case report shows that these lesions can become dysplastic.
\end{abstract}

Key words: Brunner's gland hamartomas, MUC5, polyp, duodenum

\section{INTRODUCTION}

Neoplastic growth in the small intestine is relatively infrequent. Brunner's gland hamartomas are rare duodenal tumors and an unusual cause of gastrointestinal bleeding (1). Since the first description of a Brunner's gland hamartoma in 1876 there has been controversy to the etiology and pathogenesis of this lesion. Several case reports have described these lesions as non-dysplastic and entirely benign. However, we present a case of duodenal hamartoma with an unusual inverted growth involving Brunner's gland with a dysplastic focus. This case report shows that these lesions can become dysplastic and raises the question of a malignant potential.

\section{CASE REPORT}

A 46-year-old male patient presented with epigastric discomfort. His vital signs were

\footnotetext{
*Correspondence to: Nadezhda T. Stefanova, Department of General and Clinical Pathology, Forensic Sience and Deontology, Medical University - Varna, Bulgaria, phone: 052/978 520, e-mail: nadezhda_stefanova@yahoo.com
}

normal. Physical examination results and routine laboratory tests were normal. Endoscopic examination of the upper digestive tract revealed a polyp of the duodenum, sized 8 $\mathrm{mm}$, located on the border between pars descendens and pars horizontalis, on posterior duodenal wall. Biopsy specimen was taken and the tumor was pathologically diagnosed as tubulovillous adenoma of the duodenum. Surgical operation was performed, the polyp was resected and pathology examination was done. As a whole, the mucosal surface showed a polypoid mass measuring $8 \mathrm{~cm} \times 2 \mathrm{~cm}$. Histological examination showed duodenal mucosa with hyperplasic villous structures and crypts with partial stomach epithelium, forming papillary proliferations. In the submucosa there were hyperplastic Brunner glands, some of them with cystic transformation, hyperplasia of tubules and villi lined by pseudostratified epithelium with reduction of the mucous secretion (Figure 1). Some of the epithelial cells are with moderate hyperchromasia and polymorphic nuclei. Muscularis mucosae was with aborization of adipocytes (Figure 2). In the chorion there was 
a hyperplasia of smooth muscle cells and lymphoid inflammatory infiltrate (Figure 3). Immunostaining for MUC5 was positive in the metaplastic stomach epithelium (Figure 4) MUC2- positive expression in the goblet cells. CD10 was positive in the apical part of the surface duodenal epithelium (Figure 5).

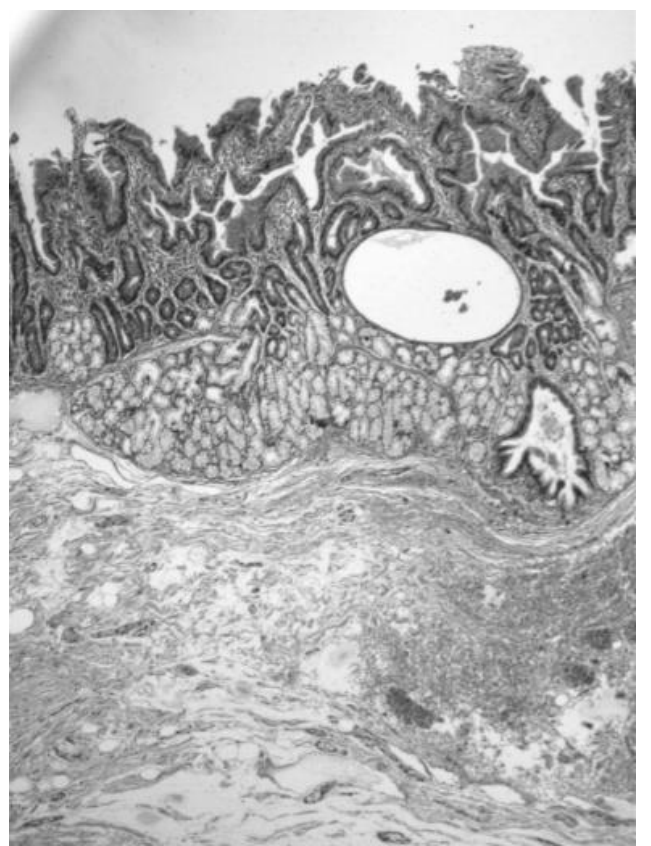

Figure 1. Part of a polyp with hyperplasia of tubules and villi, proliferation of Brunner's gland with cystic transformation. HE x 100

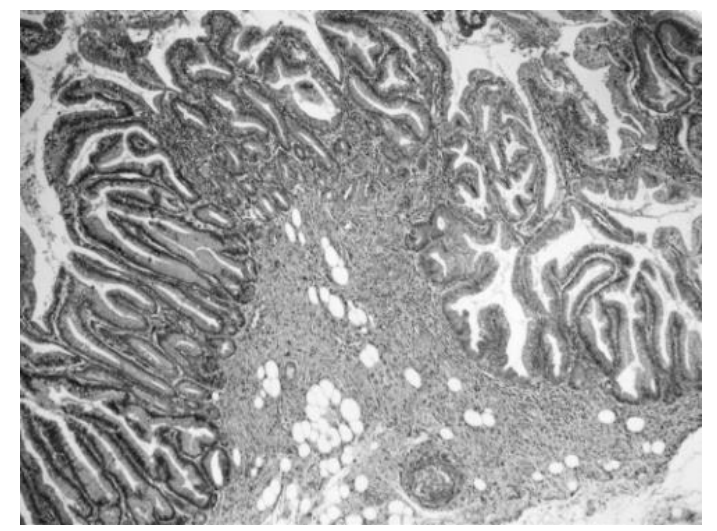

Figure 2. Part of a polyp with hyperplasia of tubules, adipocytes in muscularis mucosa. HE $\mathrm{x}$ 100 ;

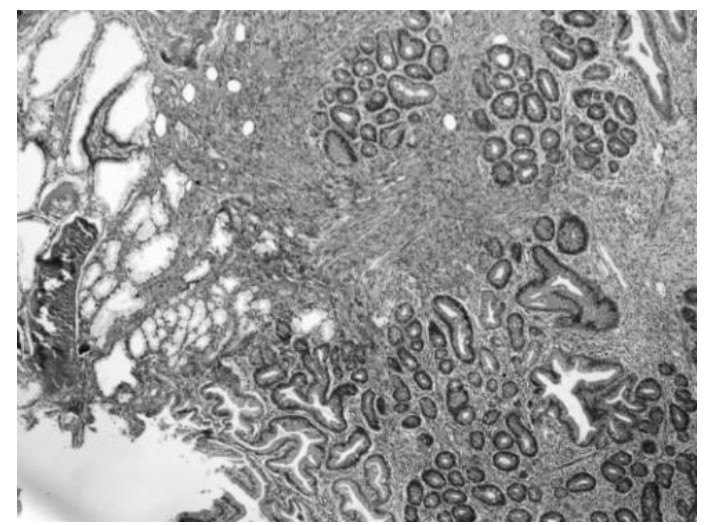

Figure 3. Hyperplasia of smooth muscle cells and lymphoid infiltrate. HE x 100;

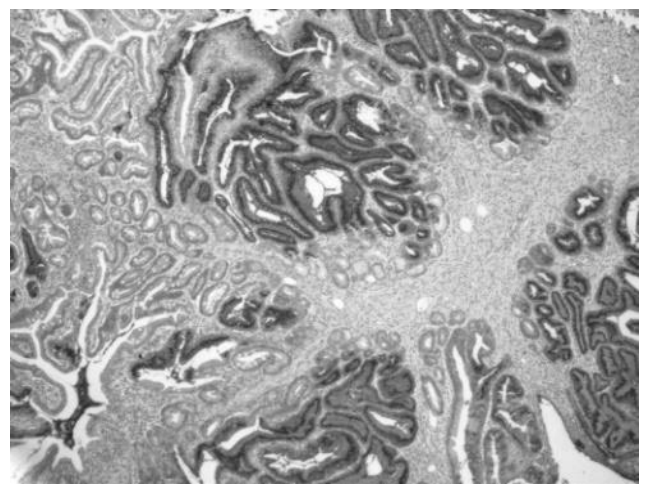

Figure 4. The immunohistochemical stain for MUC5 shows negative staining in duodenal mucosa and tubulovillous lesion, but positive in adenomatous lesion. (IHC x 100)

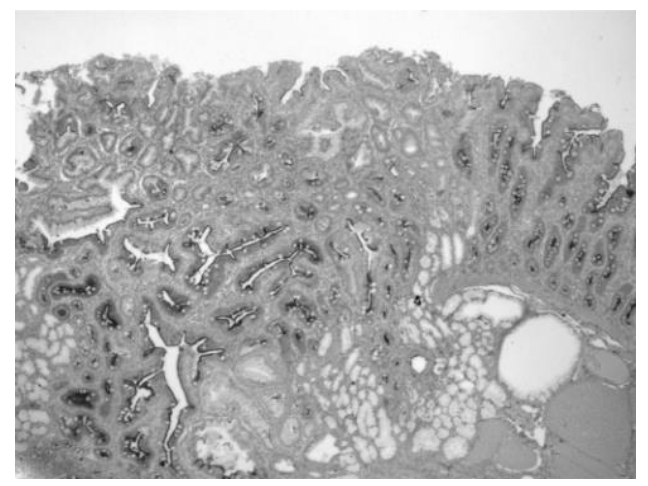

Figure 5. The duodenal surface epithelium is positive for CD10, but the Brunner's glands and adenomatous lesion are negative, (IHC x 100)

\section{DISCUSSION}

Benign tumors of the duodenum are very rare with incidence of $0.008 \%$ in a single series of 215000 autopsies (2). The nomenclature regarding Brunner's gland lesions is not well established because there is substantial degree of interchangeability among the terms "Brunner's gland hyperplasia," "Brunner's gland hamartomas", "Brunner's gland adenoma". This variation in nomenclature has led to considerable confusion in diagnosis. Brunner's gland hyperplasia has been attributed to multiple small polypoid or nodular lesions composed of excessive Brunner's gland separated by fibrous septa. Brunner's gland hamartomas has been recognized as referring to a solitary mass that contains a mixture of acini, ducts, smooth muscle, adipose tissue, and lymphoid tissue. Brunner's gland hamartomas can occasionally contain heterotopic pancreatic acini and ducts. The term "Brunner's gland adenoma", or "Brunneroma", has been applied to lesions that are histologically similar to Brunner's gland hamartomas. Because these lesions do not have features of cellular atypia , the term "Brunner's gland adenoma" has fallen out of favor (3).

The exact prevalence of Brunner's gland hamartomas $(\mathrm{BGH})$ is difficult to determine 
because of the variation in nomenclature throughout the medical literature. There is no reported race or sex predilection, and the age of presentation tends to be in the fifth and sixth decades of life $(4,5)$.

Although commonly an incidental finding, BGH have been reported to be the cause of abdominal pain, duodenal obstruction, gastrointestinal hemorrhage, duodenal intussusceptions, and obstruction of the common bile duct or pancreatic duct (6).

The pathogenesis of BGH is unknown. The most common location is the posterior wall of the duodenum (as it is in our case) near the junction of the first and second portions. The diagnosis is usually made by radiographic and endoscopic techniques. At endoscopy the classical findings are of a pedunculated polyp in the duodenal bulb, though $11 \%$ can be sessile (4), polypoid or lobulated mass, and covered with normal mucosa (7).

On gross pathological examination, Brunner's gland adenoma has a smooth surface and tends to be well-circumscribed, solitary polyps that can be sessile or pedunculated. They are pink and $\tan$ on cut surface with a lobular appearance due to fibrous septa. Histology usually reveals normal overlying mucosa and a well circumscribed lesion within the submucosa, hyperplastic Brunner's glands with a variable amount of ducts, smooth muscles, fibrous tissue, adipose tissue, and lymphocytes. Few scattered reports of duodenal adenocarcinoma arising from Brunner glands are found in the literature. Dysplasia has been shown to occur in a background of gastric metaplasia. It is postulated that Brunner gland hyperplasia and gastric metaplasia may occur secondary to duodenal mucosal injury. A recent study from Japan looked at the occurrence of dysplasia and invasive carcinoma in Brunner gland hyperplasia (8). The investigators noted dysplastic changes in $2.1 \%$ of cases of Brunner gland hyperplasia, with only $0.3 \%$ of those being invasive carcinoma.

The appearance of atypical glands with malignant potential in a Brunner adenoma has been described previously by Zanetti and Casedei, as well as Fujimaki and colleagues (9, 10).

Fujimaki and colleagues reported a case of a patient presenting with epigastric discomfort and a semi-pedunculated tumor in the duodenum found by endoscopic examination. After polypectomy, histologic examination revealed a focus of histologic and structural atypia resembling excretory ducts and not acinar cells of the tumor. Immunohistochemically, these atypical glands were strongly positive for MIB-1 (an indicator of proliferative potential) and p53 (which is negative in excretory ducts and acinar cells), indicating a possible neoplastic lesion (in situ). There have been 9 cases of adenocarcinoma arising from Brunner glands reported in the literature. Akino and colleagues (11) reported that a patient presenting with anemia had a polypoid lesion that stained positive for class 3 mucin, which is specific to Brunner glands, recognized by monoclonal antibody $\mathrm{MAB}$ HIK. The tumor was removed successfully by endoscopic mucosal resection, much like in our case. Itsuno and colleagues (12) also found a patient in whom, upon follow-up, the tumor changed from sessile to fungating and ulcerated with corresponding histologic progression from adenomatous glands to adenocarcinoma.

\section{CONCLUSION}

Brunner's gland hamartomas is uncommon benign duodenal lesion that may be either symptomatic or incidental finding. This case presents the potential for dysplasia in these lesions and raises the question of potential malignant transformation.

\section{REFERENCES}

1. Gokhale, U., Pillai, GR., Large Brunner's Gland Hamartoma: A case report. OMJ 2009, 24, 1 p 41 doi:10.5001/omj.2009.11

2. Osborne R, T.R., LownmanRM, Brunner's gland adenoma of the duodenum. Am $J$ Surg Pathol, 18: p. 689-694, 2005.

3. Nandini D.Patel, A.D.L., Anupamjit K. Mehrotra, Leslie H.Sobin, Brunner's gland hyperplasia and hamartomas:imaging features with clinicopathologic correlation. AJR, 187: p. 715-722, 2006.

4. Levine, J.A., et al., Brunner's gland hamartomas: clinical presentation and pathological features of 27 cases. Am J Gastroenterol, 90(2): p. 290-4, 1995.

5. Perez, A., et al., Benign nonampullary duodenal neoplasms. J Gastrointest Surg, 7(4): p. 536-41, 2003.

6. Stolpman, D.R., et al., Brunner's gland hamartoma: a rare cause of gastrointestinal bleeding -- case report and review of the literature. Can J Gastroenterol, 16(5): p. 309-13, 2002.

7. Kehl O, B.H., Stamm B, Amman RW, Endoscopic removal of a large, obstructing and bleeding duodenal Brunner's gland adenoma. Endoscopy, p. 231-232, 1985. 
8. Sakurai T, S.H., Honjo G, Kasyu I, Manabe $\mathrm{T}$, Gastric foveolar metaplasia with dysplastic changes in Brunner gland hyperplasia: possible precursor lesions for Brunner gland adenocarcinoma. Am J Surg Pathol, (29): p. 1442-1448, 2005.

9. Zanetti G, C.G., Brunner's gland hamartoma with incipient ductal malignancy. Report of a case. Tumori. (67): p. $75-78,1981$.

\section{STEFANOVA N., et al.}

10.Fujimaki E, N.S., Sugai T, Takeda Y, Brunner's gland adenoma with a focus of p53-positive atypical glands. Gastroenterol, (35): p. 155-158, 2000.

11.Akino K, K.Y., Ueno A, et al, Carcinoma of duodenum arising from Brunner's gland. J Gastroenterol, (37): p. 293-296, 2002.

12.Itsuno M, M.K., Omagari K, et al, Carcinoma of duodenal bulb arising from the Brunner's gland. Gastroenterol Jpn, (28): p. 118-125, 1993. 